\title{
Covalently interconnected transition metal dichalcogenide networks via defect engineering for high-performance electronic devices
}

Stefano Ippolito ${ }^{\mathrm{a}}$, Adam G. Kelly ${ }^{\mathrm{b}}$, Rafael Furlan de Oliveira ${ }^{\mathrm{a}}$, Marc-Antoine Stoeckel ${ }^{\mathrm{a}}$, Daniel Iglesias $^{a}$, Ahin Roy ${ }^{c}$, Clive Downing ${ }^{c}$, Zan Bian ${ }^{d}$, Lucia Lombardi ${ }^{d}$, Yarjan Abdul Samad ${ }^{d}$, Valeria Nicolosi ${ }^{c}$, Andrea C. Ferrari ${ }^{d}$, Jonathan N. Coleman ${ }^{b}$, Paolo Samorì ${ }^{a, *}$

a Université de Strasbourg, CNRS, ISIS UMR 7006, 8 allée Gaspard Monge, F-67000 Strasbourg, France

${ }^{b}$ School of Physics, Centre for Research on Adaptive Nanostructures and Nanodevices (CRANN) and Advanced Materials and Bioengineering Research (AMBER), Trinity College Dublin, Dublin 2, Ireland

${ }^{c}$ School of Chemistry, Centre for Research on Adaptive Nanostructures and Nanodevices (CRANN) and Advanced Materials and Bioengineering Research (AMBER), Trinity College Dublin, Dublin 2, Ireland

${ }^{d}$ Cambridge Graphene Centre, Cambridge University, $9 \mathrm{JJ}$ Thomson Avenue, Cambridge CB3 OFA, United Kingdom

* Corresponding author: samori@unistra.fr

Solution-processed semiconducting transition metal dichalcogenides (TMDs) are at the centre of an ever-increasing research effort in printed (opto)electronics. However, device performance is limited by structural defects resulting from the exfoliation process and poor inter-flake electronic connectivity. Here, we report a new molecular strategy to boost the electrical performance of TMD-based devices via the use of dithiolated conjugated molecules, to simultaneously heal sulfur vacancies in solutionprocessed transition metal disulfides $\left(\mathrm{MS}_{2}\right)$ and covalently bridge adjacent flakes, thereby promoting percolation pathways for the charge transport. We achieve a reproducible increase by one order-ofmagnitude in field-effect mobility ( $\left.\mu_{\mathrm{FE}}\right)$, current ratios (loN / loFF), and switching times (Ts) of liquid-gated transistors, reaching $10^{-2} \mathrm{~cm}^{2} \mathrm{~V}^{-1} \mathrm{~s}^{-1}, 10^{4}$, and $18 \mathrm{~ms}$, respectively. Our functionalization strategy is an universal route to simultaneously enhance the electronic connectivity in $\mathrm{MS}_{2}$ networks and tailor on demand their physicochemical properties according to the envisioned applications. 
Solution-processed layered materials have a wide-ranging portfolio of physicochemical properties, whose inherent features make them prime candidates for low-cost and scalable applications in (opto)electronics, (photo)catalysis, (bio)sensing, and biomedicine ${ }^{1,2,3,4}$. Much work has been done on the production and isolation of solution-processed semiconducting transition metal dichalcogenides (TMDs) by scalable methods ${ }^{5,6,7}$. Liquid-phase exfoliation (LPE) is the main route to attain high concentration and high volume TMD dispersions ${ }^{8,9}$, where bulk crystals are dispersed and exfoliated in a specific solvent via a mechanical energy transfer that overcomes the Van der Waals interactions within the layered structures. The high throughput achieved by LPE promotes the use of TMDs in many different applications, exploiting pristine or hybrid materials in the form of dispersions, coatings, and thin-films produced by diverse deposition techniques including inkjet printing, spray coating, roll-to-roll, drop-casting, etc ${ }^{10,4,11,7}$.

Although LPE provides the best trade-off amongst cost, purity, yield, etc. ${ }^{12,11}$, it has some limitations when the final application concerns (opto-)electronics, where structural defects in the materials play a detrimental role ${ }^{1,13}$. One of the most widely employed LPE methods makes use of a tip horn sonicator that peels layered materials apart, thanks to vibrational and cavitation forces that arise from the generation and propagation of transverse waves within the solvent ${ }^{14}$. Consequently, the formation and implosion of cavitation bubbles generates energetic shock waves that induce local temperature and pressure conditions sufficient to peel individual layers off the bulk structure, with critical influence on their ultimate lateral size ${ }^{15}$. This energetic exfoliation procedure results in a mild formation of new defects, as well as major propagation of inherent bulk defects in the exfoliated layers. Supported by thermodynamic considerations, zero-dimensional defects are the most abundant stoichiometric deficiencies in TMDs, especially chalcogen vacancies that are mainly located at the flake edges and whose formation energy is a few $\mathrm{eV}(\sim 2 \mathrm{eV}$ in the case of sulfur vacancies $)^{16,17}$. These structural defects strongly affect the electronic properties of solutionprocessed TMDs, with detrimental effects on the electrical performance of related devices ${ }^{18,19}$.

Many groups developed molecular strategies to tune the physicochemical properties of solutionprocessed TMDs and overcome the aforementioned limitations, enlarging their range of applicability in electronics and optoelectronics ${ }^{20,21,22}$. In the case of electronic applications based on individual 
flakes, a promising strategy exploits thiolated molecular systems to heal sulfur vacancies $\left(V_{S}\right)$ in transition metal disulfides $\left(\mathrm{MS}_{2}\right)$, thereby restoring the material pristine crystal structure and enhancing its electrical properties ${ }^{23,24}$. Nevertheless, in thin-film TMD-based devices, an additional and limiting factor related to the inter-flake electrical resistance emerges, resulting in a significant hindrance of charge carrier transport ${ }^{25,26}$. This represents a major bottleneck in the development of solution-processed TMD-based optoelectronics, especially in large-area and high-performance device applications.

Here, we report a molecular strategy to simultaneously heal $V_{S}$ in solution-processed $M_{2}(M=$ Mo, W, and $\mathrm{Re}$ ) and increase the inter-flake electronic connectivity by means of dithiolated molecular systems. Using $\pi$-conjugated dithiolated molecules (HS-R-SH), we prove via diverse multiscale analysis the simultaneous: i) healing of $\mathrm{V}_{\mathrm{S}}$ to restore the $\mathrm{MS}_{2}$ crystal structure and decrease the related stoichiometric deficiencies acting as charge scattering centres, ii) the covalent bridging of adjacent flakes, resulting in an enhanced charge carrier transport through an interconnected network. We investigate and capitalize on the in-situ functionalization approach of TMDs, exposing the inorganic materials to molecular linkers just after their deposition on a substrate. This is crucial for the formation of long-range pathways which exhibit superior charge transport characteristics, likewise the bridging of disordered regions in conjugated polymer chains ${ }^{27}$.

Such an approach represents an innovative and universal functionalization method capable of improving the performance of devices based on solution-processed $\mathrm{MS}_{2}$ for large-area electronic applications. We apply this strategy in liquid-gated thin-film transistors (LG-TFTs) fabricated by dropcasting $\mathrm{MS}_{2}$ dispersions onto $\mathrm{SiO}_{2} / \mathrm{Si}$ substrates pre-patterned with interdigitated gold electrodes (IDEs), followed by exposure to aromatic and conjugated 1,4-benzenedithiol (BDT) molecules. This boosts the characteristics of $\mathrm{MS}_{2}$-based LG-TFTs by one order-of-magnitude, leading to state-ofthe-art electrical performance characterized by competing field-effect mobilities $\left(\mu_{\mathrm{FE}}\right)$ and $\mathrm{l}_{\mathrm{ON}} / \mathrm{l}_{\mathrm{OFF}}$, along with the fastest switching speed reported to date for devices of this $\mathrm{kind}^{28}$. Improved water stability and mechanical robustness are other unique features exhibited by the covalently bridged $\mathrm{MS}_{2}$ networks. 


\section{Device fabrication and in-situ functionalization.}

$\mathrm{MS}_{2}$ colloidal dispersions (inks), with $\mathrm{M}=\mathrm{Mo}, \mathrm{W}$ and $\mathrm{Re}$, are produced and characterized prior to their use in devices (see Supplementary Sections 1 and 2). $\mathrm{MS}_{2}$ inks are then drop-cast onto $\mathrm{SiO}_{2} / \mathrm{Si}$ substrates with $2.5 \mu$ m-spaced Au IDEs for TFT measurements. The ink deposition is performed on the substrate placed onto a $110^{\circ} \mathrm{C}$ heated hot plate to assist the solvent (2-propanol) evaporation and the elimination of humidity traces during casting (Fig. 1a). Morphological characterizations (SEM and AFM) of the deposited materials show a large $(\mu \mathrm{m})$-scale uniform coverage of the electrodes (important to enable charge percolation pathways), thickness $=700 \pm 100 \mathrm{~nm}$, and average rootmean-square roughness $R_{r m s}=95 \pm 10 \mathrm{~nm}$ over a $25 \mu \mathrm{m}^{2}$ area (Supplementary Fig. 3a).

In our work, $\mathrm{MS}_{2}$ networks are formed by bridging adjacent flakes and taking advantage of the higher defect density at the edge sites with respect to basal planes, as confirmed by high-angle annular dark-field scanning transmission electron microscopy investigation (STEM) (Supplementary Fig. 2). More specifically, the $\mathrm{MS}_{2}$ thin films are functionalized in a $\mathrm{N}_{2}$-filled glovebox exploiting a $50 \mathrm{mM}$ saturated solution of BDT in anhydrous hexane to promote the formation of a covalentlylinked $\mathrm{MS}_{2}$ networks. The whole BDT solution preparation (powder weighing and dissolution) is carried out under $\mathrm{N}_{2}$-controlled atmosphere to avoid thiol oxidation reactions induced by impurities ${ }^{29}$. The coated slides are soaked in BDT solution at room temperature for $24 \mathrm{~h}$ inside a sealed container, followed by rinsing in hexane, and annealing onto a hot plate at $90^{\circ} \mathrm{C}$ for $30 \mathrm{~min}$. No significant morphological variations are detected after thiol exposure, and the network features remain similar to those of the pristine films (Supplementary Fig. 3b). The functionalization process is designed to simultaneously heal $\mathrm{V}_{\mathrm{S}}$ in $\mathrm{MS}_{2}$ films and covalently bridge adjacent flakes, thereby promoting their modification at the molecular level (Fig. 1b).

\section{Multiscale characterization of $\mathrm{MS}_{2}$ networks.}

We assess the effects of in-situ functionalization with BDT molecules by independent multiscale techniques. XPS measurements provide evidence for $\mathrm{MS}_{2}$ chemical functionalization, as illustrated in Fig. 2a by the S2p high-resolution spectra of drop-cast solution-processed molybdenum disulfide $\left(\mathrm{MoS}_{2}\right.$ ) before (as film) and after (as network) BDT treatment. The $\mathrm{MoS}_{2} \mathrm{~S} 2 \mathrm{p}$ spectrum displays two 
main peaks at $\sim 162.3$ and $\sim 163.5 \mathrm{eV}$, assigned to the $S 2 \mathrm{p}_{3 / 2}$ and $S 2 \mathrm{p}_{1 / 2}$ components $^{30}$, respectively. An additional component can be deconvoluted at $\sim 161.5 \mathrm{eV}$ and ascribed to defects, e.g. vacancy neighbouring $S$ atoms ${ }^{30,31}$. Such a minority component at lower binding energies is due to the charge localized on $S$, that, once $S$ is desorbed, can be redistributed on the first neighbouring atoms, enhancing Coulomb screening ${ }^{30}$. The substroichiometric $\mathrm{MoS}_{2-\mathrm{x}}$ component at $\sim 161.5 \mathrm{eV}$ is related to unsaturated $S$ ligands $^{31}$, such as $V_{S}$, and decreases from $8.0 \pm 0.5 \%$ to $5.0 \pm 0.5 \%$ upon BDT treatment, proving how the exposure to thiolated molecules leads to a decrease of chalcogen vacancy defects in solution-processed $\mathrm{MoS}_{2}$. Since different $\mathrm{S}$ ligands have minimal differences in binding energies, their identification in XPS spectra is not always straightforward, and most literature focuses just on $\mathrm{MoS}_{2}$ (see Supplementary Section 4 for further information on XPS data analysis).

a)

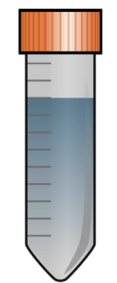

$\mathrm{MS}_{2}$ ink
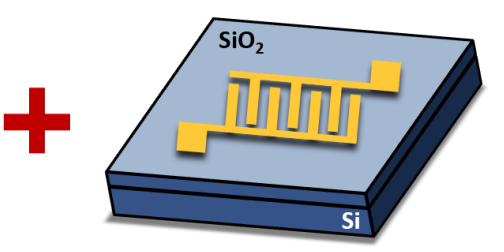

Pre-patterned Au IDEs

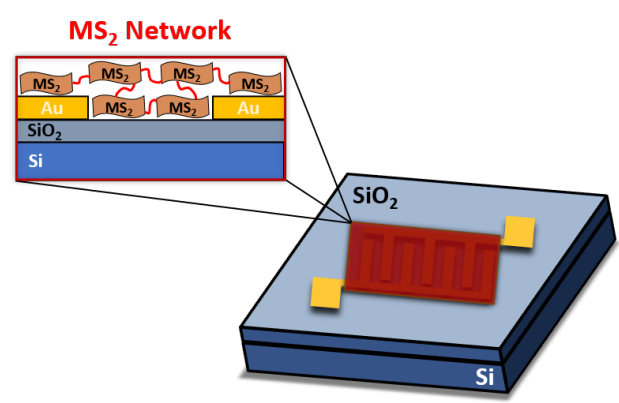

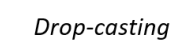

on hotplate $\left(110^{\circ} \mathrm{C}\right)$

Solution of 1,4-Benzenedithiol (BDT) in hexane Soaking (24h)

1. Solvent rinse 2. Annealing $\left(90^{\circ} \mathrm{C}\right)$

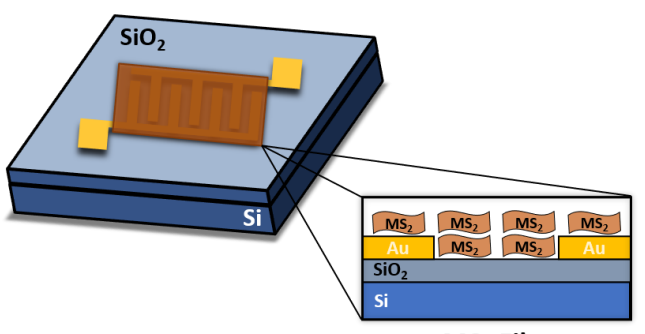

$\mathrm{MS}_{2}$ Film
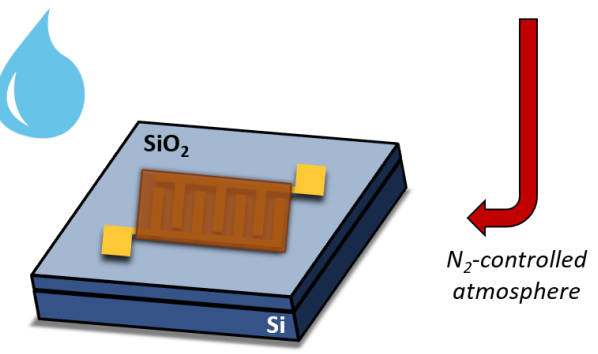

b)
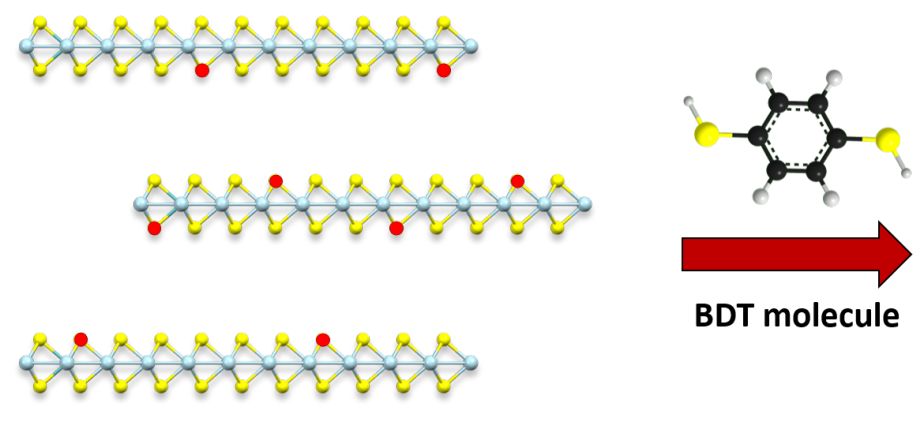

BDT molecule

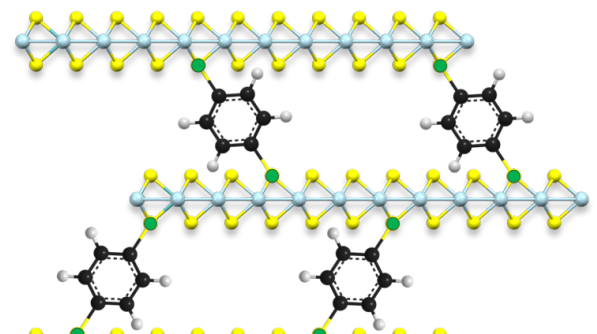

$$
\text { = Mo-Atom = S-Atom } \bullet=\text { S-Vacancy } \bullet=\text { S-Healing }
$$


Figure 1 | Functionalization strategy to produce covalently interconnected $\mathbf{M S}_{\mathbf{2}}$ networks. a, illustration of ink deposition and in-situ functionalization to produce $\mathrm{MS}_{2}$ networks via BDT treatment. $\mathbf{b}$, Sketch of $\mathrm{V}_{\mathrm{s}}$ healing mechanism in $\mathrm{MoS}_{2}$ films by means of dithiolated molecules and related inter-flake networking.

Raman spectra of $\mathrm{MoS}_{2}$ pristine films and networks (Fig. 2b) show no major differences, suggesting that the functionalization process does not damage the flakes. The full width at half maximum (FWHM) of both $E^{1}{ }_{2 g}$ and $A_{1 g}$ peaks shows a narrowing of $\sim 10 \%$ upon thiol exposure, as well as small blue shift and increase of the $E^{1}{ }_{2 g} / A_{1 g}$ intensity ratio (see Supplementary Section 5). This is consistent with a reduction in defect density and suppression of defect-activated modes ${ }^{32,33}$, endorsing the healing of $V_{S}$ by thiolated molecules. An extended and rigorous statistical Raman analysis of $\mathrm{MoS}_{2}$ films and networks is provided in Supplementary Section 5.

a)
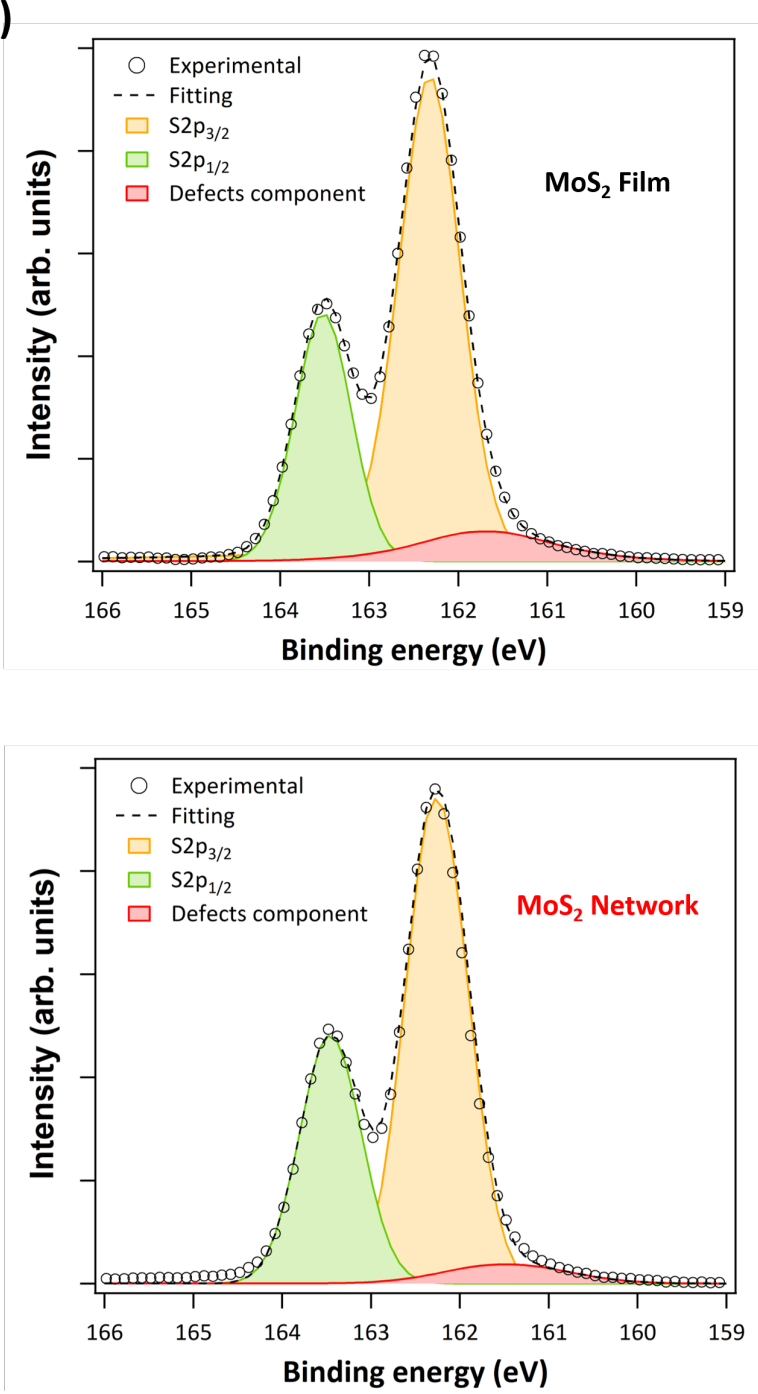

b)

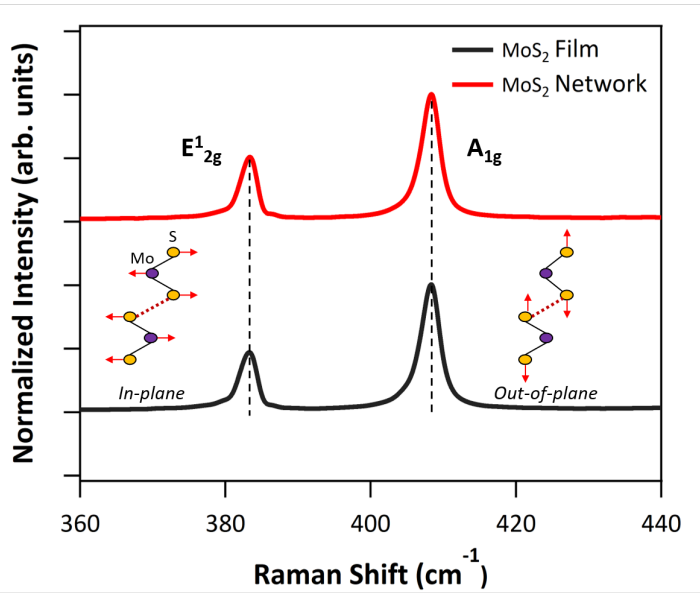

c)
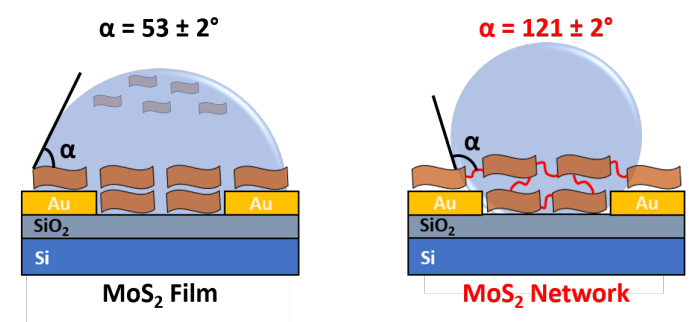

d)

Film detachment

No film detachment
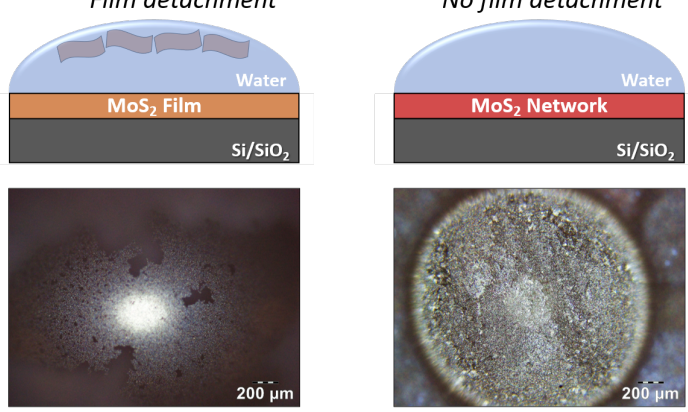
Figure 2 | Characterization of $\mathrm{MS}_{2}$ films and networks. a, High-resolution S2p XPS spectra for $\mathrm{MoS}_{2}$ films (top) and networks (bottom). b, Raman spectra of $\mathrm{MoS}_{2}$ films (black) and networks (red), highlighting the two main $E_{2 g}^{1}$ and $A_{1 g}$ peaks related to in-plane and out-of-plane vibrations, respectively. $\mathbf{c}$, Sketch of water contact angle results for $\mathrm{MoS}_{2}$ films (left) and networks (right). d, Schematics (top) and optical images (bottom) showing the different water stability for $\mathrm{MoS}_{2}$ films (left) and networks (right).

An evidence of the network formation comes from the distinctive characteristics and macroscopic properties of BDT-treated $\mathrm{MS}_{2}$ samples. Pristine $\mathrm{MoS}_{2}$ films on electrode-free $\mathrm{SiO}_{2} / \mathrm{Si}$ substrates exhibit a hydrophilic behaviour, with an average static water contact angle (WCA) of $53 \pm 2^{\circ}$ in agreement with literature ${ }^{34}$, whereas small fluctuations around these numbers depend on material growth, exfoliation, and deposition techniques ${ }^{35}$. Upon functionalization, the $\mathrm{MoS}_{2}$ networks show a strong hydrophobic behaviour characterized by an average WCA of $121 \pm 2^{\circ}$ (Fig. 2c), where the free aromatic and non-polar ring of BDT molecules remain exposed to the samples' surface increasing its hydrophobicity (see Supplementary Section 6). The network formation in BDTfunctionalized $\mathrm{MoS}_{2}$ samples improves the material stability in water (Fig. 2d and Supplementary Fig. 12). For $\mathrm{MoS}_{2}$ pristine films we observe detachment and floating of the material exposed to water, while for $\mathrm{MoS}_{2}$ networks the sample integrity is preserved. The solvation process is hindered within the $\mathrm{MoS}_{2}$ network (less soluble than isolated free single flakes), consistent with the covalent interconnectivity promoted by dithiolated linkers. Such a feature is of primary importance for the fabrication of robust devices operating in aqueous environment ${ }^{2}$. An additional evidence of network formation comes from the ex-situ functionalization of $\mathrm{MoS}_{2}$ flakes in solution ${ }^{36,37}$, where the bridging process induced by BDT linkers compromises the colloidal stability and undermines the electrical performance of the corresponding LG-TFTs (see Supplementary Section 9) ${ }^{38,39}$. All the abovementioned features of the networks cannot be achieved by using monothiolated functionalizing molecules (thiophenol, TP), that are unlikely to bridge adjacent flakes (see Supplementary Section 6). Improved mechanical robustness was observed in $\mathrm{MS}_{2}$ networks deposited onto flexible substrates and subjected to multiple (5-10k) deformations, highlighting another advantageous effect of the covalent bridging (see Supplementary Section 6, Supplementary Fig. 13). 


\section{LG-TFTs based on $\mathrm{MS}_{2}$ films and networks.}

The covalent bridging of individual $\mathrm{MS}_{2}$ flakes with $\pi$-conjugated molecules is expected to improve the material's electrical properties, especially its electrical connectivity, where long-range electronic delocalization is advocated ${ }^{27}$. We thus investigate the performance of TFTs based on pristine $\mathrm{MoS}_{2}$ films and networks. Dielectrically-gated TFTs based on solution-processed TMDs show poor current switching $\left(\mathrm{ION}_{\mathrm{ON}} / \mathrm{I}_{\mathrm{OFF}}<10\right)^{40}$, encouraging one to focus on TFTs where the semiconductor layer is electrolytically gated by means of an ionic liquid (IL) solution (Fig. 3a), exploiting the inherent disorder and related porosity of the deposited materials. For LG-TFTs based on solution-processed TMD flakes, the liquid dielectric penetrates the internal free volume of the semiconducting material, thus gating the device volumetrically ${ }^{28}$.

a)

b)
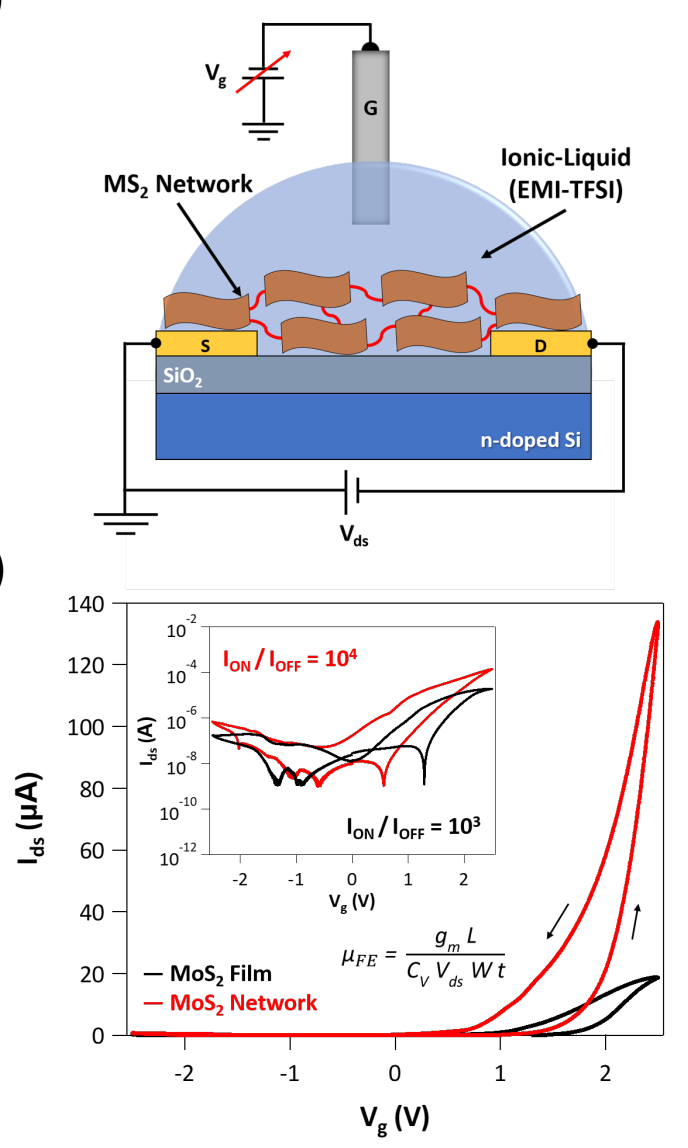

c)

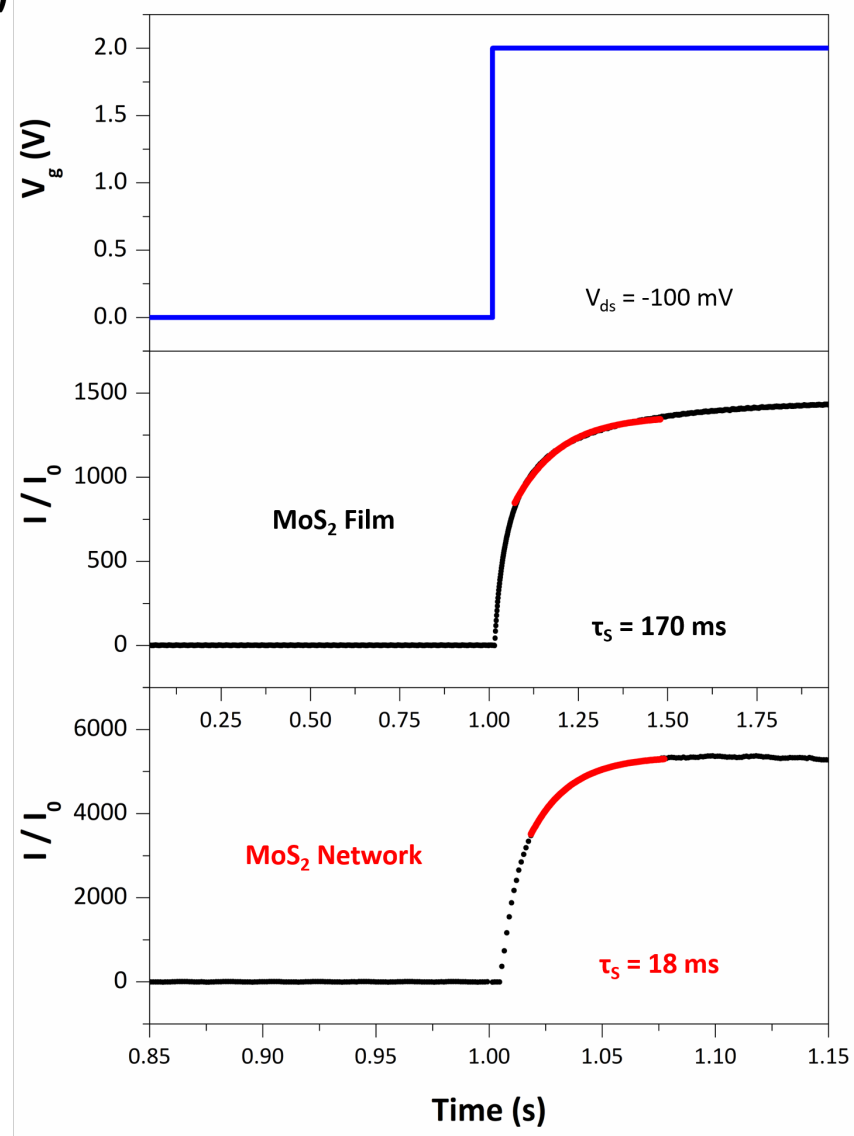

Figure 3 | Electrical properties of LG-TFTs based on MoS $_{2}$ films and networks. a, LG-TFT geometry. b, Transfer curves for $\mathrm{MoS}_{2}$ films and networks with $\mathrm{V}_{\mathrm{ds}}=-100 \mathrm{mV}$ and $\mathrm{V}_{\mathrm{g}}$ sweeping from $-2.5 \mathrm{~V}$ to $+2.5 \mathrm{~V}$. Inset: log-scale current characteristics and equation to calculate $\mu_{\mathrm{FE}}$. c, LG-TFT switching characteristics under $V_{g}$ 
step and corresponding time-dependent normalized current response $\left(\mathrm{I} / \mathrm{I}_{0}\right)$. The red line delimits the range in which a non-linear fitting can be used to extrapolate ts.

All our LG-TFT measurements are performed under $\mathrm{N}_{2}$-controlled glovebox atmosphere, to avoid side effects of environmental adsorbates, such as water and oxygen, that can induce strong pdoping $^{41}$. Fig. $3 b$ displays the transfer curves $\left(I_{d s} v s . V_{g}\right)$ of LG-TFTs based on $M_{0} S_{2}$ films and networks. Both show n-type transfer characteristics, with the latter featuring an overall superior performance. In particular, $\mathrm{MoS}_{2}$ networks exhibit higher $\mu_{\mathrm{FE}}$ up to $10^{-2} \mathrm{~cm}^{2} \mathrm{~V}^{-1} \mathrm{~s}^{-1}$ and $\mathrm{I}_{\mathrm{ON}} / \mathrm{l}_{\mathrm{OFF}}$ ratios up to $10^{4}$, one order-of-magnitude greater than pristine $\mathrm{MoS}_{2}$ films (see Supplementary Section 7 for the calculation of the device figures of merit and related statistical analysis). No significant differences in threshold voltage $\left(\mathrm{V}_{\mathrm{TH}}\right)$ are observed upon bridging of flakes, proving that BDT linkers mainly affect the conductivity of the networks in terms of $\mu_{\mathrm{FE}}$ and not the charge carrier density (doping effect) ${ }^{42}$.

Likewise, a similar outcome is observed for the switching time (Ts) of LG-TFTs based on MoS 2 films and networks, while applying a step-like $V_{g}$ stimulus and measuring the device time-dependent current response. Here, $\mathrm{Ts}_{\mathrm{s}}$ is $\sim 170 \mathrm{~ms}$ for $\mathrm{MoS}_{2}$ films and $\sim 18 \mathrm{~ms}$ for the networks (Fig. 3c), meaning that covalently interconnected systems result in one order-of-magnitude faster devices, with stateof-the-art switching performance for transistors of this $\mathrm{kind}^{28}$. The electrical characteristics and LGTFT figures of merit of other solution-processed TMDs are in the Supplementary Section 7. The reproducible 10-fold enhancement of device performance observed for $\mathrm{MS}_{2}$ networks supports the considerations envisaged for interconnected systems by m-conjugated and dithiolated linkers (Table 1), unachievable for monothiolated TP molecules, that do not allow network formation (Supplementary Fig. 20). The bridging process of adjacent flakes attained with aliphatic dithiolated molecules barely improves the electrical characteristics of $\mathrm{MoS}_{2}$ LG-TFTs, whose performance cannot rival those achieved with BDT $\pi$-conjugated linkers (Supplementary Fig. 22).

\section{Temperature-dependent electrical characteristics.}

$\mathrm{MoS}_{2}$ films and networks have also been analysed by measuring their current vs. electric field (I-E) characteristics as a function of temperature $(T)$ in high vacuum $\left(10^{-6}\right.$ Torr). The samples are prepared 
on $\mathrm{SiO}_{2} / \mathrm{Si}$ substrates with $\mathrm{Au}$ IDEs. The average field $\mathrm{E}$ is calculated from $\mathrm{V}=\mathrm{E} x d$, where $\mathrm{V}$ is the applied bias voltage and $d$ is the IDE channel distance $(2.5 \mu \mathrm{m})$. I-E relations are used with equations describing charge transport models ${ }^{43}$. The charge transport characteristics are measured with and without a back-gate voltage $\left(\mathrm{V}_{\mathrm{G}}{ }^{\text {Back }}\right)$ on the $\mathrm{n}^{++}-\mathrm{Si}$ substrate. At room temperature, and with $\mathrm{V}_{\mathrm{G}}{ }^{\mathrm{Back}}=$ 0, minor differences are found between the I-E traces of $\mathrm{MoS}_{2}$ films and networks (Fig. 4a). For T ranging from 250 to $300 \mathrm{~K}$, the current characteristics indicate Schottky emission as the dominant charge transport mechanism (Fig. 4b). The formation of a Schottky barrier (Fig. 4b inset) with height $\Phi_{\mathrm{B}}$ at the metal/MoS 2 interface was previously investigated ${ }^{44}$. From the thermionic emission formalism (Equation S4) ${ }^{43}$, we estimate the $A u / M_{0 S} \Phi_{B}=366 \pm 1 \mathrm{meV}$ for $\mathrm{MoS}_{2}$ pristine films, $\Phi_{\mathrm{B}}=285 \pm 7 \mathrm{meV}$ for $\mathrm{MoS}_{2}$ networks, and $\Phi_{\mathrm{B}}=288 \pm 16 \mathrm{meV}$ for TP-functionalized $\mathrm{MoS}_{2}$ (Supplementary Fig. 24-25). Such a reduction of $\Phi_{B}$ upon both thiol functionalization (BDT and TP) points to either a modification of the Au work-function $\left(\Phi_{\mathrm{WF}}\right)$ and/or healing of $\mathrm{Au} / \mathrm{MoS}_{2}$ interface states $^{45}$. Measurements of $\Phi_{\mathrm{WF}}$ for Au electrode surfaces by PhotoElectron Spectroscopy in Air (PESA), before and after thiol treatment, reveal a small decrease from $5.11 \pm 0.02 \mathrm{eV}$ (bare $\mathrm{Au}$ ) to $4.97 \pm 0.10 \mathrm{eV}$ and $4.93 \pm 0.07 \mathrm{eV}$ for BDT and TP, respectively (Table S6). Such a small $\Phi_{\mathrm{WF}}$ change is consistent with the passivation of Au/MoS ${ }_{2}$ interfaces states - due to the healing of $V_{S}$ in the material - as the main cause of $\Phi_{\mathrm{B}}$ reduction in thiol functionalized samples ${ }^{45}$. Such a small $\Phi_{\mathrm{WF}}$ reduction, as well as similar values found for BDT and TP-functionalized samples, cannot explain the enhanced device performance exhibited by $\mathrm{MoS}_{2}$ networks in LG-TFTs. 
a)

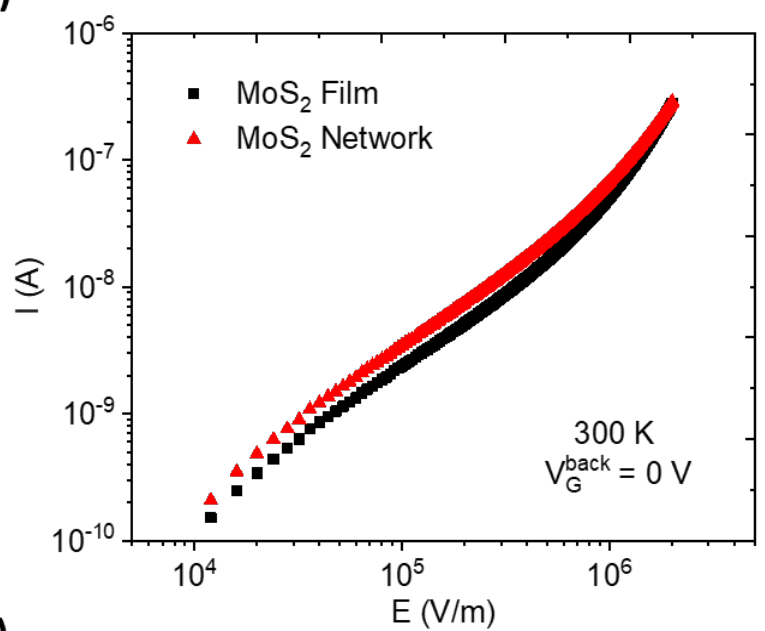

c)

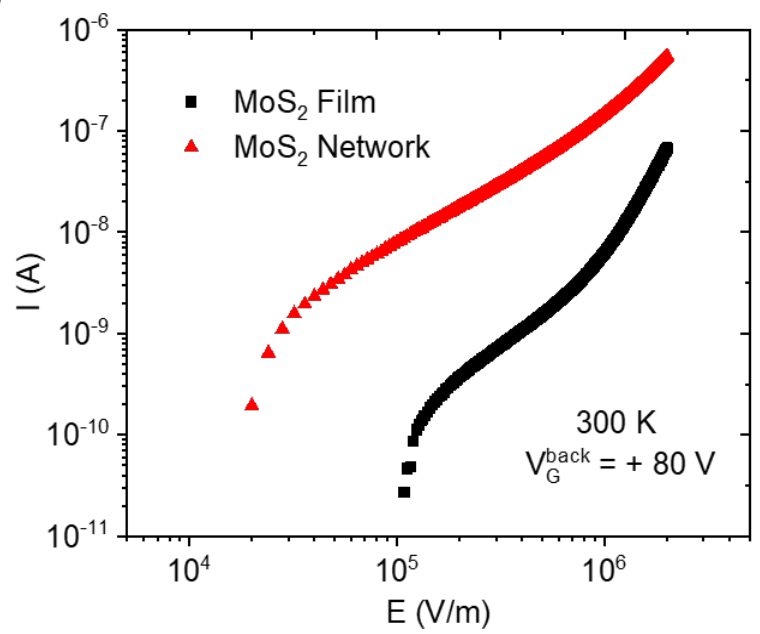

b)

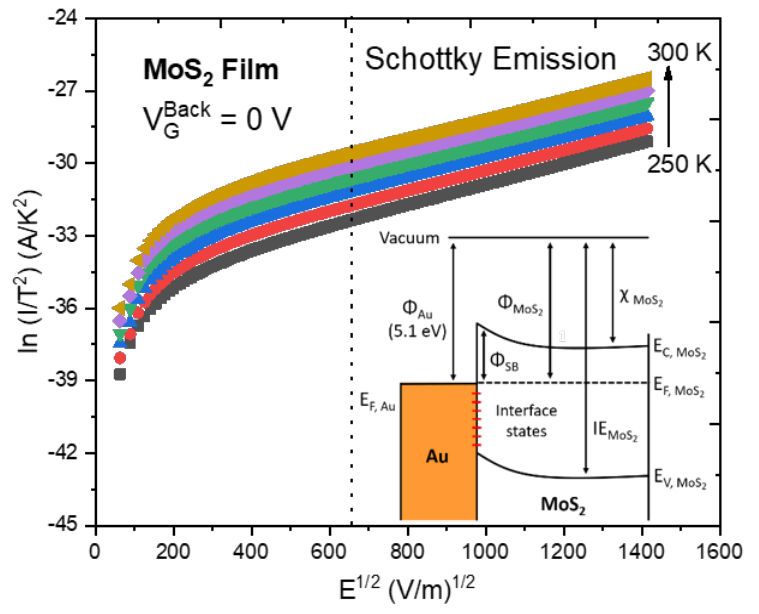

d)

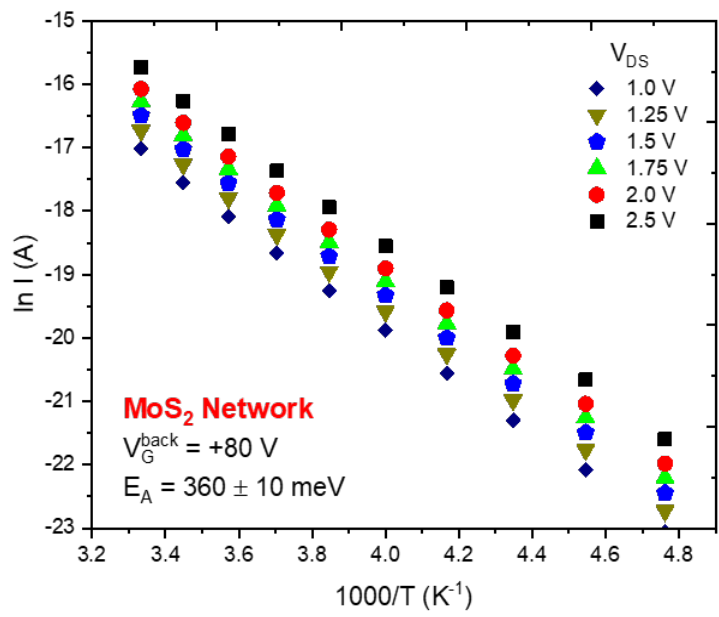

Figure 4 | Temperature-dependent electrical characteristics. a, $300 \mathrm{~K} \mathrm{I-E} \mathrm{curves} \mathrm{for} \mathrm{V}_{\mathrm{G}}{ }^{\mathrm{Back}}=0$ for $\mathrm{MoS}_{2}$ films (black) and networks (red). b. Schottky plot for $\mathrm{MoS}_{2}$ film for $\mathrm{V}_{\mathrm{G}}{ }^{\text {Back }}=0 \mathrm{~V}$. Inset: Band diagram for $\mathrm{Au} / \mathrm{MoS}_{2}$ Schottky barrier interface. c, $300 \mathrm{~K} \mathrm{I-E} \mathrm{characteristic} \mathrm{with} \mathrm{for} \mathrm{V}_{\mathrm{G}}{ }^{\mathrm{Back}}=+80 \mathrm{~V}$ in $\mathrm{MoS}_{2}$ films (black) and networks (red). d, Arrhenius plot for $\mathrm{MoS}_{2}$ network for $\mathrm{V}_{\mathrm{G}}{ }^{\text {Back }}=+80 \mathrm{~V}$.

As for LG-TFTs (Fig. 3), the superior electrical performance of $\mathrm{MoS}_{2}$ networks arise when the Schottky barrier is attenuated by the application of a $\mathrm{V}_{\mathrm{G}}{ }^{46}$. Fig. $4 \mathrm{c}$ plots the room-temperature I-E characteristics of samples under high $\mathrm{V}_{\mathrm{G}}{ }^{\mathrm{Back}}=+80 \mathrm{~V}$. A $\sim 10$-fold current difference is observed for $\mathrm{MoS}_{2}$ networks when compared to films, especially at intermediate fields $\left(E=10^{5}-10^{6} \mathrm{~V} / \mathrm{m}\right), a$ condition that mimics the LG-TFT operating parameters $\left(E \sim 10^{5} \mathrm{~V} / \mathrm{m}\right)$. For $\mathrm{T}=250-300 \mathrm{~K}$ and $E=1 \mathrm{MV} / \mathrm{m}, \mathrm{MoS}_{2}$ films and networks have a thermally-activated current response (Fig. $4 \mathrm{~d}$ and Supplementary Fig. 26) with significantly different activation energies $\left(E_{A}\right)$, i.e. $512 \pm 12 \mathrm{meV}$ and $360 \pm 10 \mathrm{meV}$, respectively. $E_{A}$ of hundreds of meV reflect the energy necessary to overcome the inter-flake barriers in $\mathrm{MoS}_{2}$ systems ${ }^{26}$, rather than low-energy (tens of meV) intra-flake conduction 
states $^{26}$. By sweeping $V_{G}{ }^{\text {Back }}$ from -60 to $+60 \mathrm{~V}$, the $T$-dependent charge carrier mobility $\mu(T)$ of films and networks follows an Arrhenius relation, with lower $E_{A}$ for networks (Supplementary Fig. 27). Hence, as inter-flake processes appear to be the limiting factor for charge transport within TMD thinfilms ${ }^{41}$, a reduced $E_{A}$ points out an improved bulk connectivity among adjacent flakes (network formation). $E_{A}$ for TP-functionalized samples (500 $\pm 1 \mathrm{meV}$ ) are only slightly smaller than the values found for pristine $\mathrm{MoS}_{2}$ films, revealing a reduction of trap states caused by the $V_{S}$ healing mechanism, without any further improvement due to the inter-flake connectivity (see Supplementary Section 8).

\section{Conclusions}

We reported a universal and simple route to produce covalently interconnected TMD networks by exploiting defect engineering in solution-processed layered materials. We used $\pi$-conjugated dithiolated molecules to bridge adjacent $\mathrm{MS}_{2}$ flakes, forming networks characterized by substantially different physicochemical properties (improved electrical characteristics, water stability, and mechanical robustness). The bridging of neighbouring flakes at the molecular level improves the charge transport across the network, thereby leading to superior device performances. LG-TFTs show a reproducible one order-of-magnitude increase in the main figures of merit, leading to stateof-the-art field-effect mobility $\left(10^{-2} \mathrm{~cm}^{2} \mathrm{~V}^{-1} \mathrm{~s}^{-1}\right)$ and IoN / IofF ratio $\left(10^{4}\right)$, along with the fastest switching time (18 ms) reported for devices of this kind ${ }^{28,47}$. Our findings pave the way for the development of high-performance, large-area and printed electronics based on solution-processed TMDs. The network formation results in water-stable and mechanically robust $\mathrm{MS}_{2}$-based devices, that could be exploited in (bio)sensing ${ }^{48}$, (photo) catalisyis $^{2}$, and flexible optoelectronics ${ }^{49}$. Ultimately, with an appropriate molecular design of the bridging linkers, one might endow the TMD networks with diverse functionalities, tuning the final properties on demand according to the final applications. 
Figure of Merit $\quad$ MoS $_{2}$ Film $\quad$ MoS $_{2}$ Network

\begin{tabular}{c|cc}
\hline$\mu_{\mathrm{FE}} / \mu_{\mathrm{FE}, \text { film }}$ & 1 & $10 \pm 1$ \\
$\mathrm{I}_{\mathrm{ON}} / \mathrm{I}_{\mathrm{OFF}}$ & $10^{3}$ & $10^{4}$ \\
$\mathrm{~V}_{\mathrm{TH}}$ & $(1.9 \pm 0.1) \mathrm{V}$ & $(1.8 \pm 0.1) \mathrm{V}$ \\
$\mathrm{TS}$ & $(170 \pm 5) \mathrm{ms}$ & $(18 \pm 2) \mathrm{ms}$
\end{tabular}

Table 1 | Main figures of merit for $\mathrm{MoS}_{2}$ film and network-based electrical devices. Upon exposure to BDT and network formation, LG-TFTs exhibit a reproducible one order-of-magnitude enhancement in the main device figures of merit, with minimal changes in $\mathrm{V}_{\mathrm{TH}}$. 


\section{Methods}

Device preparation. Bottom-contact $\mathrm{SiO}_{2} / \mathrm{n}^{++}$-Si substrates $(15 \mathrm{~mm} \times 15 \mathrm{~mm}$, Fraunhofer IPMS, Dresden, Germany) are used. The substrates consist of thermally grown $\mathrm{SiO}_{2}(230 \mathrm{~nm}$ thick $)$ having IDEs (30 nm thick Au onto $10 \mathrm{~nm}$ ITO adhesion layer) spaced $2.5 \mu \mathrm{m}$, yielding a channel widthlength (W/L) ratio $\sim 4000$. Prior to use, the substrates are cleaned by ultrasonication in acetone and 2-propanol (10 min each), and dried under $\mathrm{N}_{2}$ flow afterwards.

The films are moved in a $\mathrm{N}_{2}$-filled glovebox for the following functionalization steps: i) sample immersion in a $50 \mathrm{mM}$ saturated solution BDT in anhydrous hexane for $24 \mathrm{~h}$ inside a sealed container, ii) spin-rinsing with anhydrous hexane $(5 \mathrm{ml}, 4000 \mathrm{rpm}$, acceleration $4000 \mathrm{rpm}$ $\left.\mathrm{s}^{-1}, 60 \mathrm{~s}\right)$ and iii) annealing at $90^{\circ} \mathrm{C}$ for $30-45 \mathrm{~min}$.

Electrical characterization. The LG-TFT performances are evaluated by their transfer characteristics ( $I_{d s}$ vs. $V_{g}$ ), using a $\mathrm{Pt}$ wire as the gate electrode and a droplet of 1-ethyl-3methylimidazolium bis(trifluoromethylsulfonyl) imide EMI-TFSI as IL gate dielectric. The source-drain current $\left(I_{d s}\right)$ is recorded while sweeping gate voltage $\left(V_{g}\right)$ from -2.5 to $+2.5 \mathrm{~V}$, at source-drain voltage $V_{d s}=-0.1 \mathrm{~V}$. The current-electric field traces $\left(I_{d s}-E\right)$ are measured for $T=80-300 K$, in an Oxford Instruments Optistat $D N-V$ cryostat, for $V_{G}{ }^{B a c k}=0$ and $+80 \mathrm{~V}$. All electrical measurements are carried out in dark and under $\mathrm{N}_{2}$-controlled atmosphere using a Keithley 2636A SourceMeter unit. In order to rely on a strong statistical analysis, nearly 60 identical devices were produced and subjected to the different electrical characterizations.

Further details about the materials, characterization techniques and data treatment can be found in the Supplementary Information file.

\section{Data availability}

The data that support the findings of this study are available from the corresponding author upon reasonable request. 


\section{References}

1. Wang, Q. H., Kalantar-Zadeh, K., Kis, A., Coleman, J. N. \& Strano, M. S. Electronics and optoelectronics of two-dimensional transition metal dichalcogenides. Nat. Nanotechnol. 7, 699-712 (2012).

2. Voiry, D., Yang, J. \& Chhowalla, M. Recent strategies for improving the catalytic activity of 2D TMD nanosheets toward the hydrogen evolution reaction. Adv. Mater. 28, 6197-6206 (2016).

3. Chen, Y., Tan, C., Zhang, H. \& Wang, L. Two-dimensional graphene analogues for biomedical applications. Chem. Soc. Rev. 44, 2681-2701 (2015).

4. Ferrari, A. C. et al. Science and technology roadmap for graphene, related two-dimensional crystals, and hybrid systems. Nanoscale 7, 4598-4810 (2015).

5. Manzeli, S., Ovchinnikov, D., Pasquier, D., Yazyev, O. V. \& Kis, A. 2D transition metal dichalcogenides. Nat. Rev. Mater. 2, 1-15 (2017).

6. Han, J. H., Kwak, M., Kim, Y. \& Cheon, J. Recent advances in the solution-based preparation of twodimensional layered transition metal chalcogenide nanostructures. Chem. Rev. 118, 6151-6188 (2018).

7. Backes, C. et al. Production and processing of graphene and related materials. $2 D$ Mater. 7, 022001 (2020).

8. O'Neill, A., Khan, U. \& Coleman, J. N. Preparation of high concentration dispersions of exfoliated MoS $_{2}$ with increased flake size. Chem. Mater. 24, 2414-2421 (2012).

9. Yao, Y. et al. High-concentration aqueous dispersions of $\mathrm{MoS}_{2}$. Adv. Funct. Mater. 23, 3577-3583 (2013).

10. Bonaccorso, F., Bartolotta, A., Coleman, J. N. \& Backes, C. 2D-Crystal-based functional inks. Adv. Mater. 28, 6136-6166 (2016).

11. Bonaccorso, F. et al. Production and processing of graphene and 2d crystals. Materials Today 15, 564589 (2012).

12. Raccichini, R., Varzi, A., Passerini, S. \& Scrosati, B. The role of graphene for electrochemical energy storage. Nat. Mater. 14, 271-279 (2015).

13. Jariwala, D., Sangwan, V. K., Lauhon, L. J., Marks, T. J. \& Hersam, M. C. Emerging device applications for semiconducting two-dimensional transition metal dichalcogenides. ACS Nano 8, 1102-1120 (2014).

14. Ciesielski, A. \& Samorì, P. Graphene via sonication assisted liquid-phase exfoliation. Chem. Soc. Rev. 43, 381-398 (2014).

15. Backes, C. et al. Equipartition of energy defines the size-thickness relationship in liquid-exfoliated nanosheets. ACS Nano 13, 7050-7061 (2019). 
16. Tsai, C. et al. Electrochemical generation of sulfur vacancies in the basal plane of $\mathrm{MoS}_{2}$ for hydrogen evolution. Nat. Commun. 8, 15113 (2017).

17. Komsa, H.-P. et al. Two-dimensional transition metal dichalcogenides under electron irradiation: defect production and doping. Phys. Rev. Lett. 109, 035503 (2012).

18. McDonnell, S., Addou, R., Buie, C., Wallace, R. M. \& Hinkle, C. L. Defect-dominated doping and contact resistance in MoS 2 . ACS Nano 8, 2880-2888 (2014).

19. Nicolosi, V., Chhowalla, M., Kanatzidis, M. G., Strano, M. S. \& Coleman, J. N. Liquid exfoliation of layered materials. Science 340, (2013).

20. Voiry, D. et al. Covalent functionalization of monolayered transition metal dichalcogenides by phase engineering. Nat. Chem. 7, 45-49 (2015).

21. Ippolito, S., Ciesielski, A. \& Samorì, P. Tailoring the physicochemical properties of solution-processed transition metal dichalcogenides via molecular approaches. Chem. Commun. 55, 8900-8914 (2019).

22. Bertolazzi, S., Gobbi, M., Zhao, Y., Backes, C. \& Samorì, P. Molecular chemistry approaches for tuning the properties of two-dimensional transition metal dichalcogenides. Chem. Soc. Rev. 47, 6845-6888 (2018).

23. Schmidt, H., Giustiniano, F. \& Eda, G. Electronic transport properties of transition metal dichalcogenide field-effect devices: surface and interface effects. Chem. Soc. Rev. 44, 7715-7736 (2015).

24. Sim, D. M. et al. Controlled doping of vacancy-containing few-layer $\mathrm{MoS}_{2}$ via highly stable thiol-based molecular chemisorption. ACS Nano 9, 12115-12123 (2015).

25. Yu, X., Prévot, M. S. \& Sivula, K. Multiflake thin film electronic devices of solution processed 2D MoS 2 enabled by sonopolymer assisted exfoliation and surface modification. Chem. Mater. 26, 5892-5899 (2014).

26. Zeng, X., Hirwa, H., Metel, S., Nicolosi, V. \& Wagner, V. Solution processed thin film transistor from liquid phase exfoliated $\mathrm{MoS}_{2}$ flakes. Solid-State Electron. 141, 58-64 (2018).

27. Noriega, R. et al. A general relationship between disorder, aggregation and charge transport in conjugated polymers. Nat. Mater. 12, 1038-1044 (2013).

28. Kelly, A. G. et al. All-printed thin-film transistors from networks of liquid-exfoliated nanosheets. Science 356, 69-73 (2017).

29. Schilter, D. Thiol oxidation: a slippery slope. Nat. Rev. Chem. 1, 1-1 (2017).

30. Donarelli, M., Bisti, F., Perrozzi, F. \& Ottaviano, L. Tunable sulfur desorption in exfoliated MoS $_{2}$ by means of thermal annealing in ultra-high vacuum. Chem. Phys. Lett. 588, 198-202 (2013). 
31. McIntyre, N. S., Spevack, P. A., Beamson, G. \& Briggs, D. Effects of argon ion bombardment on basal plane and polycrystalline MoS$_{2}$. Surf. Sci. 237, L390-L397 (1990).

32. Mignuzzi, S. et al. Effect of disorder on Raman scattering of single-layer MoS2. Phys. Rev. B 91, 195411 (2015).

33. Bae, S. et al. Defect-induced vibration modes of Ar+-Irradiated MoS 2 . Phys. Rev. Applied 7, 024001 (2017).

34. Park, S. Y. et al. Highly selective and sensitive chemoresistive humidity sensors based on $\mathrm{rGO} / \mathrm{MoS}_{2}$ van der Waals composites. J. Mater. Chem. A 6, 5016-5024 (2018).

35. Chow, P. K. et al. Wetting of mono and few-layered $\mathrm{WS}_{2}$ and $\mathrm{MoS}_{2}$ films supported on $\mathrm{Si} / \mathrm{SiO}_{2}$ Substrates. ACS Nano 9, 3023-3031 (2015).

36. Nguyen, E. P. et al. Electronic tuning of 2D MoS 2 through surface functionalization. Adv. Mater. 27, 6225$6229(2015)$.

37. Chou, S. S. et al. Ligand conjugation of chemically exfoliated MoS 2 . J. Am. Chem. Soc. 135, 4584-4587 (2013).

38. Kim, J. et al. Direct exfoliation and dispersion of two-dimensional materials in pure water via temperature control. Nat. Commun. 6, 1-9 (2015).

39. Graetzel, M., Janssen, R. A. J., Mitzi, D. B. \& Sargent, E. H. Materials interface engineering for solutionprocessed photovoltaics. Nature 488, 304-312 (2012).

40. Li, J., Naiini, M. M., Vaziri, S., Lemme, M. C. \& Östling, M. Inkjet printing of $\mathrm{MoS}_{2}$. Adv. Funct. Mater. 24, 6524-6531 (2014).

41. Li, S.-L., Tsukagoshi, K., Orgiu, E. \& Samorì, P. Charge transport and mobility engineering in twodimensional transition metal chalcogenide semiconductors. Chem. Soc. Rev. 45, 118-151 (2015).

42. Wang, Y., Gali, S. M., Slassi, A., Beljonne, D. \& Samorì, P. Collective dipole-dominated doping of monolayer $\mathrm{MoS}_{2}$ : orientation and magnitude control via the supramolecular approach. Adv. Funct. Mater. 30, 2002846 (2020).

43. Chiu, F.-C. A Review on conduction mechanisms in dielectric films. Adv. Mater. Sci. Eng. 2014, 1-18 (2014).

44. Lee, K. et al. Electrical characteristics of molybdenum disulfide flakes produced by liquid exfoliation. Adv. Mater. 23, 4178-4182 (2011).

45. Sze, S. M. \& Ng, K. K. Physics of semiconductor devices. John Wiley \& Sons (2006).

46. Vladimirov, I. et al. Bulk transport and contact limitation of $\mathrm{MoS}_{2}$ multilayer flake transistors untangled via temperature-dependent transport measurements. Phys. Status Solidi A 212, 2059-2067 (2015). 
47. Higgins, T. M. et al. Electrolyte-gated n-type transistors produced from aqueous inks of $\mathrm{WS}_{2}$ Nanosheets. Adv. Funct. Mater. 29, 1804387 (2019).

48. Anichini, C. et al. Chemical sensing with 2D materials. Chem. Soc. Rev. 47, 4860-4908 (2018).

49. Akinwande, D., Petrone, N. \& Hone, J. Two-dimensional flexible nanoelectronics. Nat. Commun. 5, 5678 (2014). 


\section{Acknowledgements}

We acknowledge funding from European Commission through the Graphene Flagship, the ERC Grants SUPRA2DMAT (GA-833707), FUTURE-PRINT (GA-694101), Hetero2D, GSYNCOR, the EU Grant Neurofibres, the Agence Nationale de la Recherche through the Labex projects CSC (ANR10-LABX-0026 CSC) and NIE (ANR-11-LABX-0058 NIE) within the Investissement d'Avenir program (ANR-10-120 IDEX-0002-02), the International Center for Frontier Research in Chemistry (icFRC), EPSRC Grants EP/K01711X/1, EP/K017144/1, EP/N010345/1, EP/L016057/1, and the Faraday Institution. The HAADF-STEM characterization was carried out in the Advanced Microscopy Laboratory (Dublin), a Science Foundation Ireland (SFI) supported centre.

\section{Author contributions}

S.I. and P.S. conceived the experiments and designed the study. A.G.K., Z.B., L.L., Y.A.S., A.C.F. and J.N.C. produced the raw materials and characterized them by spectroscopic and electrochemical techniques. S.I. designed and performed the multiscale characterizations on the final functionalized materials. R.F.O and M.A.S. designed and performed the charge carrier transport measurements and studies. D.I. carried out the NMR measurements and analysis. A.R., C.D. and V.N. designed and performed the HAADF-STEM investigations. All authors discussed the results and contributed to the interpretation of data. S.I., R.F.O, and P.S. co-wrote the paper with input from all co-authors.

\section{ORCID}

Stefano Ippolito: 0000-0002-6906-3961

Adam G. Kelly: 0000-0002-6070-7070

Rafael Furlan de Oliveira: 0000-0001-8980-3587

Marc-Antoine Stoeckel: 0000-0002-6410-4058

Daniel Iglesias: 0000-0002-1998-0518

Ahin Roy: 0000-0002-9515-2562

Clive Downing: 0000-0002-9209-0036 
Zan Bian: 0000-0002-1659-8460

Lucia Lombardi: 0000-0002-6438-2971

Yarjan Abdul Samad: 0000-0001-9323-4807

Valeria Nicolosi: 0000-0002-7637-4813

Andrea C. Ferrari: 0000-0003-0907-9993

Jonathan N. Coleman: 0000-0001-9659-9721

Paolo Samorì: 0000-0001-6256-8281

\section{Competing financial interests}

The authors declare no competing financial interests.

\section{Additional information}

Supplementary information is available in the online version of the paper. Reprints and permission information is available online at www.nature.com/reprints. Correspondence and requests for materials should be addressed to P.S. 